\title{
Le plan de communication : définir et organiser votre stratégie de communication de Thierry Libaert, Paris, Dunod, 2000, 179 p., 149 F
}

Isabelle Cousserand

\section{OpenEdition}

Journals

Édition électronique

URL : http://journals.openedition.org/communicationorganisation/2539

DOI : 10.4000/communicationorganisation.2539

ISSN : 1775-3546

Éditeur

Presses universitaires de Bordeaux

Édition imprimée

Date de publication : 1 mai 2001

ISSN : 1168-5549

Référence électronique

Isabelle Cousserand, «Le plan de communication : définir et organiser votre stratégie de communication de Thierry Libaert, Paris, Dunod, 2000, 179 p., $149 \mathrm{~F} »$, Communication et organisation [En ligne], 19 | 2001, mis en ligne le 27 mars 2012, consulté le 30 avril 2019. URL : http:// journals.openedition.org/communicationorganisation/2539; DOI : 10.4000/ communicationorganisation.2539

Ce document a été généré automatiquement le 30 avril 2019.

(c) Presses universitaires de Bordeaux 


\title{
Le plan de communication : définir et organiser votre stratégie de communication de Thierry Libaert, Paris, Dunod, 2000, 179 p., 149 F
}

\author{
Isabelle Cousserand
}

1 Si les ouvrages à vocation méthodologique et professionnelle sont légions, beaucoup se contentent d'esquisser une opérationnalité élémentaire quelque peu superficielle et éphémère. Face aux enjeux épineux soulevés par l'audit de communication et sous-tendus par le plan de communication, nombre de manuels font figure de pansements.

2 Parce que l'on ne peut transiger avec les pratiques, Thierry Libaert est exigeant. Son parcours allie l'expérience de la fonction communication à celle des missions d'enseignement et de recherche universitaires. Face à la multiplicité et à la subtilité des rouages d'une politique de communication rigoureuse et cohérente, le chargé de mission ou le "dircom» est ici positionné au cœur d'un dispositif complexe, dont la pièce maîtresse est le plan de communication. L'aborder de manière professionnelle et pédagogique, rigoureuse et créative, conceptuelle et concrète, tel est le pari réussi de ce livre.

3 Comme l'indique le sous-titre, l'ouvrage se divise en deux parties et rappelle les dimensions incontournables qui concourent à l'élaboration et à la mise en œuvre d'une stratégie de communication externe.

4 La première, intitulée "Concevoir le plan de communication", se compose de trois chapitres : le plan dans la communication de l'entreprise, les préalables et les différentes formes. L'auteur définit ce qu'est le plan, ce qu'il sous-tend, ce qu'il recouvre.

5 La seconde, intitulée « Réaliser le plan de communication ». est traitée en quatre points. Dans un premier temps, une vingtaine de pages sont consacrées à l'audit, travail de diagnostic qui précède tout "plan de com» digne de ce nom. À plusieurs reprises, l'auteur rappelle cette impérieuse nécessité: "si l'audit forme le socle du plan de 
communication, son absence ou ses imperfections ne pourront amener qu'une stratégie bancale» ou encore "sans audit rigoureux, il est rare que la communication soit pertinente ». Thierry Libaert développe ensuite la définition d'objectifs précis et la mise en place de méthodes appropriées, puis l'adéquation cibles-moyens-messages, et clôture par le pilotage et le suivi du plan. La seconde partie spécifie donc les éléments fondamentaux de la démarche, sa mise en œuvre et son suivi.

6 Formes, enjeux, objectifs, réalisations et incidences du plan de communication externe sont passés au crible de l'expérience d'un homme de terrain, qui a le souci de la rigueur. Le cheminement méthodologique est utile, nécessaire, indispensable. L'ensemble est étayé par un certain nombre de figures, schémas ou tableaux. De multiples exemples et cas d'entreprise récents, les témoignages de professionnels enrichissent cet ouvrage accessible, clair et concis. L'auteur nous montre combien les dimensions opérationnelles sont riches d'enseignements, bien au-delà des cadres, des règles et des recettes quelquefois agités pour tenter de faire illusion ou masquer les faiblesses de certains choix.

7 Les étudiants ont désormais la chance de faire leurs gammes accompagnés d'un traité fiable, qui caractérise et illustre les grandes étapes de la démarche ${ }^{1}$. Les professionnels pourront s'y ressourcer pour peaufiner leurs actions et nourrir une réflexion sur le champ des pratiques. Quant aux enseignants, l'occasion leur est donnée de dépoussiérer leurs classiques.

\section{NOTES}

1. À cet égard, nous leur conseillons vivement la lecture complémentaire et tout aussi incontournable de l'ouvrage de Bernard Dagenais. Le plan de communication : l'art de séduire ou de convaincre les autres. Sainte Foy. Les Presses de l'Université de Laval, 1998, 370 p., 195 F., dont le n - 14 de la revue s'est fait l'écho. 